\title{
Ancestry-inspired genomic health
}

\begin{abstract}
A solution to screening for recessive heritable disorders and identifying genetic influences on common diseases is to be found in the history of one of the world's most populous regions. Large South Asian populations are a mosaic of smaller populations, many of which have founder effects as extreme as those in the European isolates that first inspired genetic medicine.
\end{abstract}

$D$

avid Reich, Kumarasamy Thangaraj and colleagues (p 1403) have now identified shared genome fragments that are identical by descent (IBD) in the genotypes of thousands of people from India, Pakistan, Bangladesh, Sri Lanka and Nepal and used these to quantify the founder effect in hundreds of distinctive populations. The origins of the founder effects are likely different for each population, but for many South Asian populations there is a common, shared history, according to demographic modeling of their genotypes. After a period of admixture between Ancestral North Indians and Ancestral South Indians 1,900-4,200 years ago (Am. J. Hum. Genet. 93, 422-438, 2013), the main demographic pattern has been for the resulting small populations throughout South Asia to marry within their group. Expansion of a population descended from a small group leaves characteristic signatures on the population's haplotype diversity and allele frequencies. In particular, variants causing recessive genetic disorders will become more common and easier to detect in a population with a strong founder effect. Large founder populations are also of particular interest for genetic investigation of common disease predisposition, as gene knockouts and deleterious alleles will occur at elevated population frequencies and rarer neutral alleles will be less prevalent. More signal, less noise.

As the authors point out, knowing the ancestral history of an indi- vidual with a recessive disorder within a recognized founder population allows a more efficient search for others sharing a disease-causing mutation or risk genotype that would otherwise be too rare to provide statistical evidence. As for prevention of genetic diseases, screens for recessive variants that are prevalent in groups with strong founder effects can be combined with appropriate community-based premarital counseling. Because their method filtered out the very long chromosome segments shared by the genomes of second cousins and more closely related individuals, the authors did not take the effects of any consanguinity into account in this study. Founder effects of this magnitude readily yield recessive variants in the absence of consanguinity. However, if consanguinity were present, it would act to increase the number of homozygous individuals affected by recessive-disease-causing variants, further facilitating gene discovery.

In addition to welcoming these translational opportunities, the journal is enthusiastic about the identification of a number of large groups with distinctive admixture and allele sharing patterns that can now be compared with respect to their patterns of common disease predisposition and pharmacogenetics traits, promoting genomic medicine globally and, in particular, expanding the biomedical research capacity in this diverse, populous and rapidly developing region. 